\title{
Oncological Outcomes of Patients with Concomitant Bladder and Urethral Carcinoma
}

\author{
Georgios Gakis $^{9}$ Jason A. Efstathiou ${ }^{b}$ Siamak Daneshmand ${ }^{c}$
}

Kirk A. Keegan ${ }^{d}$ Rebecca H. Clayman ${ }^{b}$ Jan Hrbacek ${ }^{\text {e }}$ Bedeir Ali-El-Dein $^{f}$

Harras B. Zaid $^{d}$ Tina Schubert ${ }^{9}$ Johannes Mischinger ${ }^{g}$ Tilman Todenhöfer ${ }^{9}$

Sigolene Galland ${ }^{b}$ Kola Olugbade Jr. ${ }^{\text {a }}$ Michael Rink ${ }^{\text {h }}$ Hans-Martin Fritsche ${ }^{i}$

Maximilian Burger ${ }^{i}$ Sam S. Chang ${ }^{d}$ Marko Babjuk $^{e}$ George N. Thalmann $^{j}$

Arnulf Stenzl $^{g}$ Todd M. Morgan ${ }^{\text {a }}$

a Department of Urology, University of Michigan, Ann Arbor, Mich., b Massachusetts General Hospital, Harvard Medical School, Boston, Mass., 'Institute of Urology, USC/Norris Comprehensive Cancer Center, Los Angeles, Calif.,

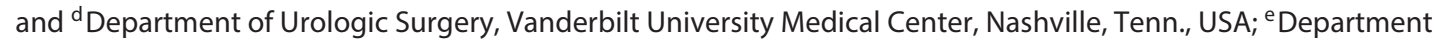
of Urology, 2nd Faculty of Medicine, Charles University, Prague, Czech Republic; ${ }^{\mathrm{f}}$ Mansoura Clinic, Urology and Nephrology Center, Mansoura, Egypt; ${ }^{9}$ Department of Urology, University of Tübingen, Tübingen, ${ }^{\text {h Department }}$ of Urology, University Medical Center Hamburg-Eppendorf, Hamburg, and 'Department of Urology, University Hospital Regensburg, Regensburg, Germany; jDepartment of Urology, University Hospital Bern, Bern, Switzerland

\section{Key Words}

Bladder cancer · Lymph node · Prognosis · Radical

cystectomy · Risk factor · Survival · Urethra

\begin{abstract}
Introduction: The study aimed to investigate oncological outcomes of patients with concomitant bladder cancer (BC) and urethral carcinoma. Methods: This is a multicenter series of 110 patients ( 74 men, 36 women) diagnosed with urethral carcinoma at 10 referral centers between 1993 and 2012. Kaplan-Meier analysis was used to investigate the impact of BC on survival, and Cox regression multivariable analysis was performed to identify predictors of recurrence. Results: Syn-
\end{abstract}

chronous BC was diagnosed in $13(12 \%)$ patients, and the median follow-up was 21 months (interquartile range 4-48). Urethral cancers were of higher grade in patients with synchronous $B C$ compared to patients with non-synchronous $B C(p=0.020)$. Patients with synchronous BC exhibited significantly inferior 3-year recurrence-free survival (RFS) compared to patients with non-synchronous BC (63.2 vs. $34.4 \%$; $p=0.026$ ). In multivariable analysis, inferior RFS was associated with clinically advanced nodal stage ( $p<0.001)$, proximal tumor location $(p<0.001)$ and synchronous BC $(p=$ 0.020). Conclusion: The synchronous presence of $B C$ in patients diagnosed with urethral carcinoma has a significant adverse impact on RFS and should be an impetus for a multimodal approach.

\section{KARGER}

E-Mail karger@karger.com

www.karger.com/uin
(C) 2016 S. Karger AG, Basel

0042-1138/16/0972-0134\$39.50/0
Georgios Gakis

Department of Urology, Eberhard-Karls University University Hospital Tübingen

Hoppe-Seyler Strasse 3, DE-72076 Tübingen (Germany)

E-Mail georgios.gakis@googlemail.com 


\section{Introduction}

According to European cancer registries, urethral carcinoma is a very rare malignancy. It accounts for well under $1 \%$ of all malignancies, with an estimated incidence of 650 new cases per year and an age-standardized ratio of approximately 2-5 per million in men and $0.6-2$ per million in women $[1,2]$.

Given the fact that tumors of the lower urinary tract may arise from different locations within the urinary tract, there is currently no data regarding the prognostic significance of concomitant bladder and urethral cancer as compared to those patients with urethral carcinoma only [3]. With regard to the significantly higher incidence of bladder cancer (BC), the question arises as to whether synchronous $\mathrm{BC}$ impacts the prognosis of patients with urethral carcinoma $[3,4]$.

In order to develop tailored treatment pathways and optimize the management of patients with urethral carcinoma [3], the effect of synchronous BC on the prognosis of patients with urethral cancer needs to be better understood. For this reason, we have assembled a multi-institutional collaborative with the aim of determining the prognostic impact of concomitant $\mathrm{BC}$ in patients diagnosed with urethral carcinoma.

\section{Patients and Methods}

This is a retrospective study representing data from 10 international academic centers. The study was approved by the independent ethics committee at Tübingen (protocol number:469/2015BO2) and participating sites. A computerized database was generated to combine the different data sets. Through regular communication with all sites, resolution of all identified anomalies was achieved before the analyses were performed. We collected data from a total of 133 consecutive patients treated for urethral carcinoma between 1993 and 2012. A total of 23 patients (17.3\%) with evidence of distant metastatic disease at initial diagnosis were excluded from the final analysis. Furthermore, patients with a diagnosis of BC prior to the detection of urethral carcinoma or those with secondary urethral recurrence after radical cystectomy were excluded from this study.

Clinical and Histologic Assessment

We assessed the following clinical and pathologic parameters: age at primary treatment, gender, clinical and pathologic tumor stage, clinical and histopathologic lymph node (LN) involvement, histologic subtype, tumor grade, lymphovascular invasion, soft-tissue surgical margin (STSM) status, tumor location (proximal vs. distal), prior history of malignancy, modality of primary treatment, perioperative chemotherapy, preoperative serum creatinine level and the presence of synchronous BC at diagnosis of urethral carcinoma.

Clinical staging was based on preoperative bimanual examination, cystoscopy with biopsy and cross-sectional imaging. Histo- logic assessment was performed at the center-specific pathology department and was based on the 1973 WHO grading system and 2002 TNM classification as approved by the AJCC [5]. The pathologic macro- and microscopic evaluation of specimens included cross-sectioning of the entire specimen with immunohistochemical staining to identify the presence of urothelial, squamous cell and adenocarcinoma or any other histological variant [6].

\section{Treatment Approach}

The overall majority of patients underwent surgery for primary treatment while only a small proportion of them were treated with radiotherapy and/or chemotherapy only (table 1). Regional LN dissection (LND) was performed at the discretion of the treating surgeon based on intraoperative findings and preoperative imaging. The level of LND was dependent on the location of the primary tumor, encompassing the inguinal LN, external and internal iliac, obturator and common iliac LN.

\section{Follow-Up}

Electronic hospital charts and physician records were reviewed to determine clinical outcomes. Due to the rarity of the disease, no standardized follow-up existed across all centers. Typically, follow-up consisted of physical examination with laboratory testing, intravenous pyelography, cystoscopy, urine cytology, urethral washings, cross-sectional imaging and bone scintigraphy, if indicated. Recurrence was defined as disease recurrence locally in the urethra and/or in LN and/or in distant organs.

\section{Statistical Analysis}

For univariate analyses, chi-square and Fisher's exact tests were used for nominal data and Student's t test for scaled data. KaplanMeier analysis with log-rank testing was employed to investigate the impact of the presence of synchronous BC on recurrence-free survival (RFS) and overall survival (OS). For determining RFS/OS, clinical outcomes were measured from the date of primary treatment to the date of first documented recurrence/death [7].

For multivariable analysis, Cox proportional hazards regression was carried out to evaluate predictors of recurrence. $p$ values are two-sided and $\mathrm{p}<0.05$ was considered significant. Statistical analysis was performed using $\mathrm{JMP}^{\circledR} 11.0$. Values are given as mean, median and interquartile range (IQR).

\section{Results}

The clinical and histopathologic tumor and patient characteristics are listed in table 1 . The histological characteristics of $\mathrm{BC}$ are listed in table 2. Primary urethral cancers were of higher grade in patients with synchronous $\mathrm{BC}$ compared to patients with non-synchronous $\mathrm{BC}$ $(p=0.020)$. No further significant differences were found between the 3 groups. In the 51 patients who underwent LND in addition to primary tumor treatment, the median number of retrieved LN was 17 (IQR 13-21).

The location of recurrences in the 110 patients is outlined in table 1 . With a median follow-up of 21 months 
Table 1. Clinical and pathologic characteristics for $\mathrm{cM} 0$ urethral carcinoma in the 110 patients according to the timing of BC diagnosis

\begin{tabular}{|c|c|c|c|c|}
\hline & No BC & Synchronous BC & Metachronous BC & $\mathrm{p}$ value \\
\hline Number of patients, \% & $96(87.2)$ & $13(11.8)$ & $1(0.9)$ & \\
\hline Gender & & & & 0.56 \\
\hline Male & $63(65.6)$ & $10(76.9)$ & 1 & \\
\hline Female & $33(34.4)$ & $3(23.1)$ & 0 & \\
\hline Age, years & & & & 0.15 \\
\hline Median & 66 & 72 & & \\
\hline IQR & $58-77$ & $65-81$ & 72 & \\
\hline cT stage & & & & 0.32 \\
\hline cTX & $1(1.0)$ & $2(15.4)$ & 0 & \\
\hline $\mathrm{cTa}$ & $12(12.5)$ & $2(15.4)$ & 1 & \\
\hline cTis & $6(6.3)$ & $0(0)$ & 0 & \\
\hline cT1 & $32(33.3)$ & $5(38.5)$ & 0 & \\
\hline cT2 & $23(24.0)$ & $3(23.1)$ & 0 & \\
\hline cT3 & $18(18.8)$ & $0(0)$ & 0 & \\
\hline cT4 & $4(4.2)$ & $1(7.7)$ & 0 & \\
\hline pT stage & & & & 0.21 \\
\hline pT0 & $1(1.1)$ & $2(15.4)$ & 0 & \\
\hline $\mathrm{pTa}$ & $12(13.5)$ & $1(7.7)$ & 0 & \\
\hline pTis & $8(9.0)$ & $1(7.7)$ & 0 & \\
\hline pTis (pu) & $3(3.4)$ & $2(15.4)$ & 0 & \\
\hline $\mathrm{p}$ Tis (pd) & $3(3.4)$ & $0(0)$ & 0 & \\
\hline pT1 & $13(14.6)$ & $1(7.7)$ & 1 & \\
\hline pT2 & $20(22.5)$ & $3(25.0)$ & 0 & \\
\hline pT3 & $16(18.0)$ & $1(7.7)$ & 0 & \\
\hline pT4 & $13(14.6)$ & $1(7.7)$ & 0 & \\
\hline cN stage & & & & 0.72 \\
\hline $\mathrm{cNX}$ & $8(8.3)$ & $2(15.4)$ & 0 & \\
\hline cNo & $76(79.2)$ & $11(84.6)$ & 1 & \\
\hline $\mathrm{cN}+$ & $12(12.5)$ & $0(0)$ & 0 & \\
\hline pN stage & & & & 0.83 \\
\hline pNX & $41(47.1)$ & $8(61.5)$ & 1 & \\
\hline pNo & $38(43.7)$ & $0(0)$ & 0 & \\
\hline $\mathrm{pN} 1$ & $2(2.3)$ & $0(0)$ & 0 & \\
\hline $\mathrm{pN} 2$ & $6(6.9)$ & $5(38.5)$ & 0 & \\
\hline Tumor grade & & & & 0.020 \\
\hline G1 & $9(9.4)$ & 0 & 0 & \\
\hline $\mathrm{G} 2$ & $25(26.0)$ & $1(7.7)$ & 0 & \\
\hline G3 & $54(56.3)$ & $10(76.9)$ & 0 & \\
\hline GX & $7(7.3)$ & $1(7.7)$ & 1 & \\
\hline Not available & $1(1.0)$ & $1(7.7)$ & 0 & \\
\hline Histology & & & & 0.67 \\
\hline UC & $41(42.7)$ & $8(61.5)$ & 1 & \\
\hline SCC & $28(29.2)$ & $2(15.4)$ & 0 & \\
\hline $\mathrm{AC}$ & $14(14.6)$ & $1(7.7)$ & 0 & \\
\hline Mixed $(\mathrm{UC} \pm \mathrm{SCC} \pm \mathrm{AC})$ & $7(7.3)$ & $1(7.7)$ & 0 & \\
\hline Leiomyosarcoma & $1(1.0)$ & $0(0)$ & 0 & \\
\hline Melanoma & $2(2.1)$ & $0(0)$ & 0 & \\
\hline Adenoid cystic carcinoma & $1(1.0)$ & $0(0)$ & 0 & \\
\hline Sarcomatoid & $0(0)$ & $0(0)$ & 0 & \\
\hline Undifferentiated & $0(0)$ & $1(7.7)$ & 0 & \\
\hline Other & $2(2.1)$ & $0(0)$ & 0 & \\
\hline Tumor location & & & & 0.18 \\
\hline Proximal & $51(53.1)$ & $10(76.9)$ & 1 & \\
\hline Distal & $45(46.9)$ & $3(23.1)$ & 0 & \\
\hline Prior history of malignancy & & & & 0.07 \\
\hline Present & $19(19.8)$ & $5(38.5)$ & 1 & \\
\hline Absent & $77(80.2)$ & $8(61.5)$ & 0 & \\
\hline
\end{tabular}


Table 1. (continued)

\begin{tabular}{|c|c|c|c|c|}
\hline & No BC & Synchronous BC & Metachronous BC & $\mathrm{p}$ value \\
\hline \multicolumn{5}{|l|}{ Per cancer entity } \\
\hline Prostate & $8(8.3)$ & $3(23.1)$ & 1 & \\
\hline Breast & $3(3.2)$ & $0(0)$ & 0 & \\
\hline Lung & $1(1.0)$ & $0(0)$ & 0 & \\
\hline Colorectal & $3(3.2)$ & $0(0)$ & 0 & \\
\hline Kidney & $1(1.0)$ & $1(7.7)$ & 0 & \\
\hline Testicular & $1(1.0)$ & $0(0)$ & 0 & \\
\hline Vulvar & $2(2.1)$ & $0(0)$ & 0 & \\
\hline Vaginal & $1(1.0)$ & $0(0)$ & 0 & \\
\hline Uterus & $1(1.0)$ & $0(0)$ & 0 & \\
\hline Oropharyngeal & $1(1.0)$ & $0(0)$ & 0 & \\
\hline Lymphoma & $0(0)$ & $1(7.7)$ & 0 & \\
\hline \multicolumn{4}{|l|}{ Pretreatment serum creatinine level, mg/dl } & 0.09 \\
\hline Median & 1 & 1.3 & 0.7 & \\
\hline Mean & 1.0 & 1.3 & & \\
\hline IQR & $0.8-1.2$ & $1.0-1.6$ & & \\
\hline \multicolumn{4}{|l|}{ Modality of primary treatment } & 0.45 \\
\hline Transurethral resection & $27(28.1)$ & $4(30.8)$ & 0 & \\
\hline Transurethral laser resection & $1(1.0)$ & $0(0)$ & 0 & \\
\hline Partial urethrectomy & $7(7.3)$ & $0(0)$ & 0 & \\
\hline Urethrectomy & $25(26.0)$ & $1(7.7)$ & 1 & \\
\hline Prostatectomy & $0(0)$ & $0(0)$ & 0 & \\
\hline Cyst(oprostat)ectomy plus urethrectomy & $29(30.2)$ & $7(53.8)$ & 0 & \\
\hline Radiotherapy & $2(2.1)$ & $0(0)$ & 0 & \\
\hline Chemotherapy & $0(0)$ & $1(7.7)$ & 0 & \\
\hline Chemoradiotherapy & $3(3.2)$ & $0(0)$ & 0 & \\
\hline Other & $2(2.1)$ & $0(0)$ & 0 & \\
\hline \multicolumn{4}{|l|}{ Perioperative chemotherapy } & 0.61 \\
\hline Neoadjuvant & $9(9.4)$ & $2(15.4)$ & 1 & \\
\hline Neoadjuvant plus adjuvant & $4(4.2)$ & $0(0)$ & 0 & \\
\hline Adjuvant & $14(14.6)$ & $3(23.1)$ & 0 & \\
\hline None & $69(71.8)$ & $8(61.5)$ & 0 & \\
\hline \multicolumn{4}{|l|}{ Location of recurrence } & 0.55 \\
\hline LN & $10(10.4)$ & $4(30.8)$ & 0 & \\
\hline Distant & $2(2.1)$ & $1(7.7)$ & 0 & \\
\hline Urethral & $16(16.7)$ & $3(23.1)$ & 0 & \\
\hline LN plus distant & $6(6.3)$ & $0(0)$ & 1 & \\
\hline LN plus urethral & $3(3.1)$ & $0(0)$ & 0 & \\
\hline Distant plus urethral & $4(4.2)$ & $0(0)$ & 0 & \\
\hline LN plus distant plus urethral & $6(6.3)$ & $0(0)$ & 0 & \\
\hline No recurrence & $49(51.0)$ & $5(38.5)$ & 0 & \\
\hline \multicolumn{4}{|l|}{ STSM } & 0.43 \\
\hline pR0 & $56(58.3)$ & $6(46.2)$ & 1 & \\
\hline $\mathrm{pR} 1$ & $9(9.4)$ & $0(0)$ & 0 & \\
\hline pR2 & $4(4.2)$ & $1(7.7)$ & 0 & \\
\hline $\mathrm{pRX}$ & $10(10.4)$ & $2(15.4)$ & 0 & \\
\hline Not available & $17(17.7)$ & $4(30.8)$ & 0 & \\
\hline \multicolumn{4}{|l|}{ Lymphovascular invasion } & 0.43 \\
\hline LV0 & $22(22.9)$ & $5(38.5)$ & 0 & \\
\hline LVI & $14(14.6)$ & $0(0)$ & 0 & \\
\hline LVX & $38(39.6)$ & $6(46.2)$ & 1 & \\
\hline Not available & $22(22.9)$ & $2(15.4)$ & 0 & \\
\hline
\end{tabular}

$\mathrm{AC}=$ Adenocarcinoma; $\mathrm{pu}=$ prostatic urethra; $\mathrm{pd}=$ prostatic ducts; $\mathrm{UC}=$ urothelial carcinoma.

No significant differences obtained when patients with metachronous disease was added to the group of patients with BC. 
Table 2. Clinical and pathologic BC characteristics in the 14 patients with urethral carcinoma

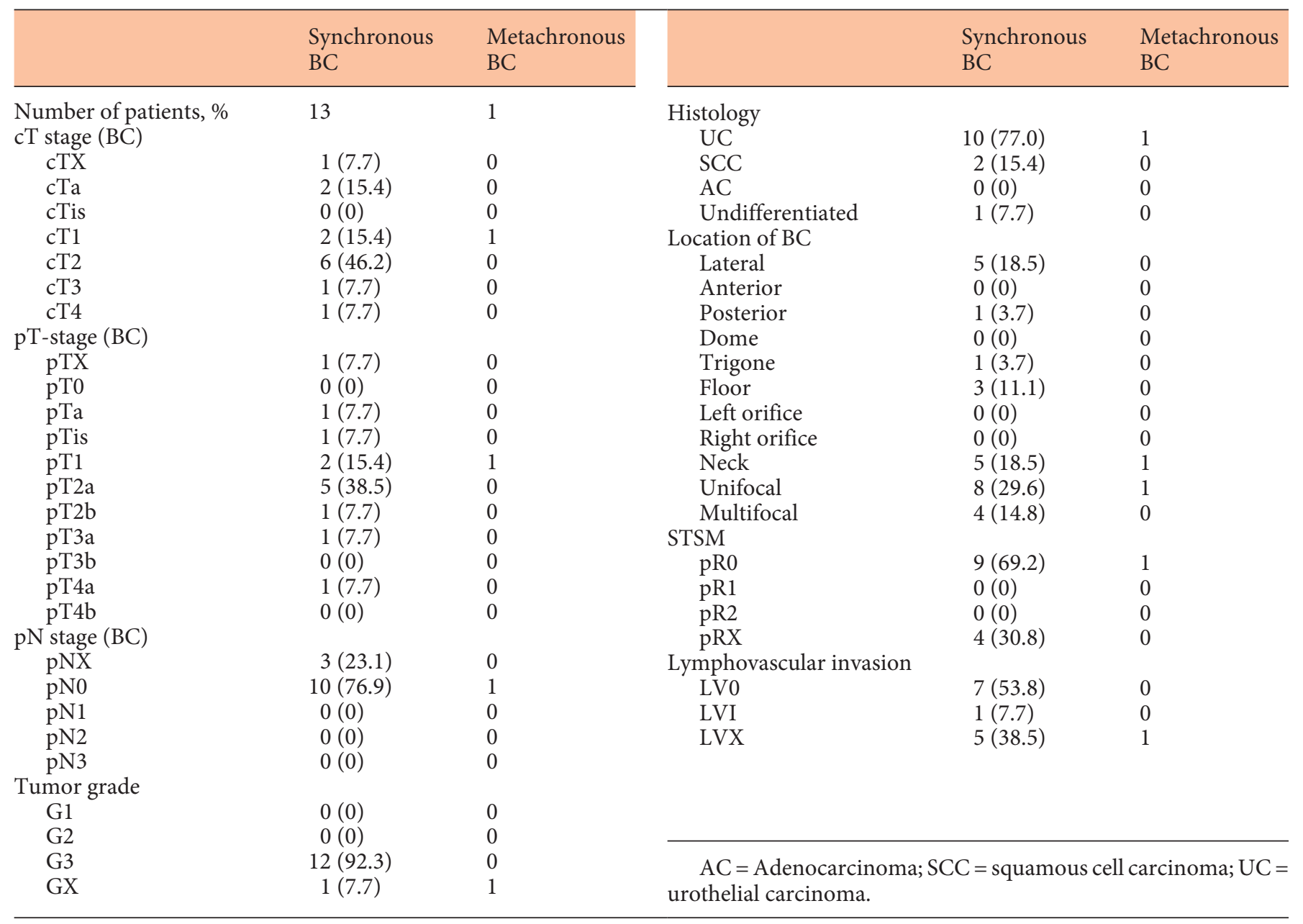

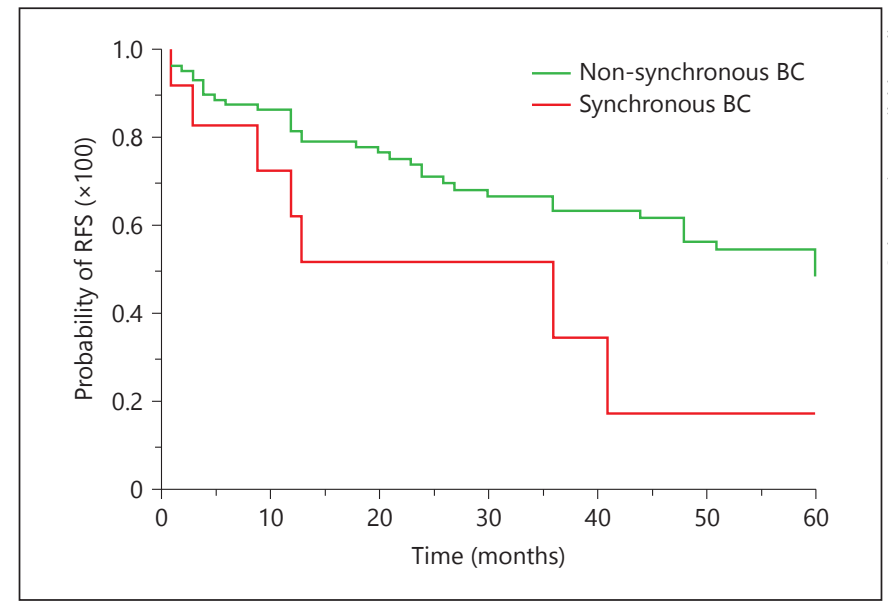

Fig. 1. Kaplan-Meier analysis for RFS in the 110 patients staged cM0 according to the presence of synchronous vs. non-synchronous/no BC $(\mathrm{p}=0.026)$. (mean 32, IQR 4-48), recurrences occurred in 56 of the 110 patients $(50.9 \%)$. The corresponding 3 - and 5 -year RFS rates were 60.5 and $45.3 \%$, respectively. A total of 26 (23.6\%) of the 110 patients died of other causes during the follow-up period, with a corresponding 3-year OS of 78.7\%. Patients with synchronous BC exhibited significantly inferior 3-year RFS compared to patients with nonsynchronous BC (63.2 vs. $34.4 \%$; $p=0.026$; fig. 1 ) with a trend toward inferior 3-year OS also approaching statistical significance ( 80.0 vs. $70.0 \%$; $=0.07$ ). In patients with synchronous $\mathrm{BC}$, receipt of perioperative chemotherapy (either given neoadjuvantly or adjuvantly) was not associated with a difference in 3 -year OS (60\%) compared to patients who did not receive chemotherapy $(47.6 \% ; \mathrm{p}=0.87)$.

In the univariate Cox regression analyses, recurrence was significantly associated with the presence of synchronous $\mathrm{BC}(\mathrm{p}=0.05)$, histopathologically advanced tumor 
Table 3. Univariate Cox regression analysis for RFS and OS in the 110 patients with cM0 primary urethral carcinoma according to clinical and pathologic tumor characteristics

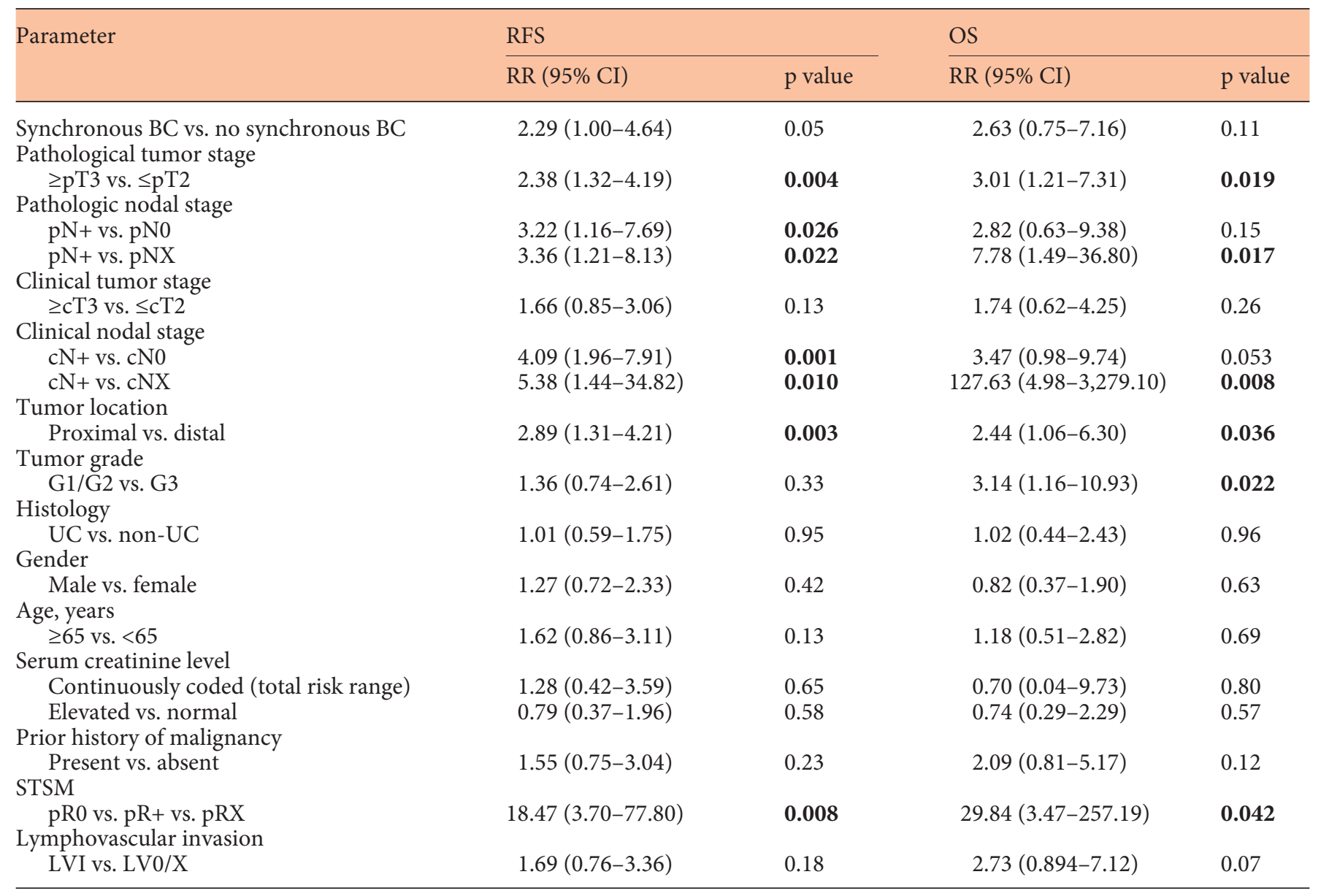

$\mathrm{RR}=$ Relative risk; $\mathrm{UC}=$ urothelial carcinoma. Bold values indicate statistically significant difference.

stage ( $\geq p$ T3; $p=0.004)$, clinically and histopathologically confirmed node-positive disease $(\mathrm{p}=0.001$ and $\mathrm{p}=$ $0.026)$, proximal tumor location $(\mathrm{p}=0.003)$ and positive STSM ( $p=0.008)$. No significant associations were found between recurrence and age, gender, clinical tumor stage, tumor grade, prior history of another malignant disease, pretreatment serum creatinine levels, lymphovascular invasion and histological subtype (table 2 ).

In the univariate analyses for OS, histopathologically advanced tumor stage $(\mathrm{p}=0.019)$, clinically and histopathologically node-positive disease $(\mathrm{cN}+$ vs. $\mathrm{cNX}, \mathrm{p}=$ $0.014 ; \mathrm{pN}+$ vs. $\mathrm{pNX}, \mathrm{p}=0.017)$, proximal tumor location $(\mathrm{p}=0.036)$, STSMs $(\mathrm{p}=0.042)$ and tumor grade $(\mathrm{p}=$ 0.022 ) were all associated with an increased risk of death. No significant associations were found between overall mortality and lymphovascular invasion $(\mathrm{p}=0.07)$, clini-

Synchronous BC and Urethral Cancer cal tumor stage, age, synchronous presence of BC, gender, prior history of malignant disease, pretreatment serum creatinine levels and histology (table 3).

In the multivariable Cox regression analysis, recurrence was significantly associated with clinically nodepositive disease and proximal tumor location (both $\mathrm{p}<$ 0.001 ), as well as the presence of synchronous BC at the time of primary treatment $(\mathrm{p}=0.020$; table 4$)$.

\section{Discussion}

Since urethral cancer is a rare tumor entity, the prognostic significance of clinical and pathologic risk factors prior to initiation of treatment is uncertain. As the incidence of $\mathrm{BC}$ is relatively much higher, the impact of 
Table 4. Multivariate analysis for predicting recurrence in the 110 patients with $\mathrm{cM} 0$ bladder and urethral carcinoma

\begin{tabular}{|c|c|c|c|c|c|c|}
\hline \multirow[t]{2}{*}{ Variable } & \multicolumn{3}{|l|}{ Full model } & \multicolumn{3}{|l|}{ Final model } \\
\hline & $\begin{array}{l}\text { regression } \\
\text { coefficient }\end{array}$ & HR (95\% CI) & $\mathrm{p}$ value & $\begin{array}{l}\text { regression } \\
\text { coefficient }\end{array}$ & $\mathrm{HR}(95 \% \mathrm{CI})$ & $\mathrm{p}$ value \\
\hline \multicolumn{7}{|l|}{ BC } \\
\hline Synchronous vs. no BC/metachronous BC & 0.795 & $4.91(1.30-17.71)$ & 0.020 & 0.383 & $2.14(1.00-4.54)$ & 0.05 \\
\hline Tumor location & & & & & & \\
\hline Proximal vs. distal & 1.094 & $8.93(3.07-33.70)$ & $<0.001$ & 0.486 & $2.64(1.45-5.05)$ & 0.001 \\
\hline Clinical nodal stage & & & & & & \\
\hline cN+ vs. cN0 vs. cNX & 1.285 & $13.08(3.40-50.61)$ & $<0.001$ & 0.926 & $6.37(2.93-13.07)$ & $<0.001$ \\
\hline Tumor grade & & & & & & \\
\hline G3 vs. G1/G2 & 0.475 & $2.59(0.93-7.58)$ & 0.068 & & & \\
\hline Pathologic tumor stage & & & & & & \\
\hline$\geq \mathrm{pT} 3$ vs. $\leq \mathrm{pT} 2$ & 0.348 & $2.00(0.73-5.34)$ & 0.17 & & & \\
\hline Lymphovascular invasion & & & & & & \\
\hline LVI vs. LV0 vs. LVX & 0.685 & $1.98(0.58-6.79)$ & 0.27 & & & \\
\hline STSMs & & & & & & \\
\hline pR+ vs. pR0 vs. pRX & 0.227 & $1.25(0.27-6.29)$ & 0.74 & & & \\
\hline
\end{tabular}

concomitant $\mathrm{BC}$ in urethral carcinoma has not been evaluated, and no clinically meaningful prognostic analyses have been reported so far. We therefore set up a unique collaborative to determine important predictors of oncologic outcomes in patients with urethral carcinoma and accrued a total of 110 cases with cM0 disease.

In the present study, only one patient developed metachronous BC 41 months after primary treatment for urethral carcinoma. For this reason, we included patients with non-synchronous $\mathrm{BC}$ in a single prognostic stratum and compared this group to patients with synchronous BC. We found that most patients with synchronous BC had high-grade urethral carcinoma and exhibited inferior RFS compared to patients with non-synchronous BC. Additionally, the majority of patients with synchronous BC exhibited histopathologically confirmed muscle invasive disease. These findings indicate that the synchronous presence of both malignant diseases exerts a markedly adverse impact on outcomes. In addition, we found that approximately one-fourth of the patients in this cohort had a history of another malignancy, with prostate cancer being the most common. However, we did not observe an adverse impact of prior malignancy on RFS or OS. As this series is retrospective, we cannot accurately adjust for any causative association between the primary treatment for these malignancies and the carcinogenesis of urethral cancer.
As would be expected, recurrence was significantly associated with advanced clinical nodal stage, advanced histopathological tumor and nodal stage, proximal tumor location, STSMs and synchronous BC. These results are in accordance with prior studies $[2,8,9]$. For the multivariable analysis, we included all significant parameters from the univariate analyses that correlated with survival. Clinical nodal stage, tumor location and presence of synchronous BC were the only parameters found to be independently associated with RFS. Importantly, we recently reported that clinical nodal stage is a strong predictor for outcomes in primary urethral cancer [10]. As the number of events was low $(n=26$ death events), we did not perform a multivariable analysis for OS.

This study has limitations inherent to its retrospective and multi-center design, which is requisite given the rarity of urethral carcinoma. Although we included patients treated at academic centers within the last 20 years, the number of included patients and follow-up period is limited due to the rarity and aggressiveness of the disease. Further potential biases include the absence of LND in approximately half of the patients and interobserver variabilities in the clinical staging and histopathologic assessment of specimens. In addition, adjustments for patient preferences, toxicities and side effects of primary treatment as well as comorbidities could not be taken into account. 
We recently reported that neoadjuvant chemo(radio) therapy also exerts a beneficial impact on outcomes in patients with clinically advanced primary urethral cancer $(\geq \mathrm{cT} 3$ and or $\mathrm{cN}+$ ) [11]. The number of patients with concomitant bladder and urethral carcinoma was rather small $(\mathrm{n}=13)$ making it difficult to draw meaningful conclusions on the role of neoadjuvant chemotherapy. However, only 2 of the patients with concomitant bladder and urethral carcinoma received neoadjuvant chemotherapy even though both malignancies were staged as muscle invasive ( $\geq \mathrm{pT} 2 \mathrm{BC}: \approx 62 \%$; $\geq \mathrm{pT} 2$ urethral carcinoma $\approx 56 \%$ ) in the majority of cases. Taking the results of the multivariable analysis into consideration, neoadjuvant chemotherapy is likely an important treatment strategy for patients with concomitant bladder and urethral carcinoma who have clinically node-positive disease and proximal urethral tumors.

As the incidence of $\mathrm{BC}$ is much higher compared to urethral cancer, one cannot exclude the possibility that primary BC developed first in the lower urinary tract which then metastasized to the urethra rather than occurring synchronously. Additionally, as this report includes different histological subtypes of urethral carcinoma, the tumor biology and treatment regimens may have impacted key outcomes. Therefore, combining all these cases into one group carries important limitations. However, population-based studies have shown that the histological subtype of urethral carcinoma does not appear to exert an impact on prognosis $[1,2,8,9]$. Nonetheless, it has to be acknowledged that urethral cancer is a rare disease for which therapeutic strategies still need to be better defined [12]. Though secondary urethral cancer after RC for BC is more frequent than concomitant urethral and $\mathrm{BC}$ it is difficult to draw meaningful conclusions for clinical practice owing to distinct differences in tumor biologic characteristics [13]. Given this, to our opinion, our study gives important insights into the clinical course of patients with urethral carcinoma which may be further used for prognostication and clinical decision-making in daily practice.

\section{Conclusions}

This is the first series that has investigated the impact of synchronous BC on outcomes of patients with urethral cancer. The findings of this study highlight the impaired prognosis of these patients and should be an impetus to consider a multimodal approach for the subgroup of patients with concomitant bladder and urethral cancer.

\section{Acknowledgments}

None.

\section{Disclosure Statement}

All authors have nothing to disclose related to the content of this study. No funding was obtained for this study.

\section{References}

$>1$ Visser O, Adolfsson J, Rossi S, Verne J, Gatta G, Maffezzini M, et al; RARECARE Working Group: Incidence and survival of rare urogenital cancers in Europe. Eur J Cancer 2012;48: 456-464.

$>2$ Swartz MA, Porter MP, Lin DW, Weiss NS: Incidence of primary urethral carcinoma in the United States. Urology 2006;68:1164-1168.

-3 Gakis G, Witjes JA, Compérat E, Cowan NC, De Santis M, Lebret T, et al: EAU guidelines on primary urethral carcinoma. Eur Urol 2013;64:823-830.

4 Stenzl A, Draxl H, Posch B, Colleselli K, Falk M, Bartsch G: The risk of urethral tumors in female bladder cancer: can the urethra be used for orthotopic reconstruction of the lower urinary tract? J Urol 1995;153(3 pt 2):950955.

5 Sobin LH, Wittekind C: TNM Classification of Malignant Tumors, ed 6. New York, WileyLiss, 2002.
-6 Shim JW, Cho KS, Choi YD, Lee DW, Han WS, et al: Diagnostic algorithm for papillary urothelial tumors in the urinary bladder. Virchows Arch 2008;452:353-362.

$>7$ Rink M, Fajkovic H, Cha EK, Gupta A, Karakiewicz PI, Chun FK, et al: Death certificates are valid for the determination of cause of death in patients with upper and lower tract urothelial carcinoma. Eur Urol 2012;61:854855.

$>8$ Derksen JW, Visser O, de la Rivière GB, Meuleman EJ, Heldeweg EA, Lagerveld BW: Primary urethral carcinoma in females: an epidemiologic study on demographical factors, histological types, tumour stage and survival. World J Urol 2013;31:147-153.

-9 Rabbani F: Prognostic factors in male urethral cancer. Cancer 2011;117:2426-2434.

10 Gakis G, Morgan TM, Efstathiou JA, Keegan KA, Mischinger J, Todenhoefer T, et al: Prognostic factors and outcomes in primary ure- thral cancer: results from the international collaboration on primary urethral carcinoma. World J Urol 2016;34:97-103.

11 Gakis G, Morgan TM, Daneshmand S, Keegan KA, Mischinger J, Todenhofer T, et al: Impact of perioperative chemotherapy on survival in patients with advanced primary urethral cancer: results of the international collaboration on primary urethral carcinoma. Ann Oncol 2015;26:1754-1759.

12 Fahmy O, Scharpf M, Fend F, Stenzl A, Gakis G: Feasibility of penis-preserving surgery for urethral melanoma: proposal for a therapeutic algorithm. Clin Genitourin Cancer 2015; 13:e411-e413.

13 Hrbáček J, Macek P, Ali-El-Dein B, Thalmann GN, Stenzl A, Babjuk M, et al: Treatment and outcomes of urethral recurrence of urinary bladder cancer in women after radical cystectomy and orthotopic neobladder: a series of 12 cases. Urol Int 2015;94:45-49. 